\title{
Time-Resolved Study of the Photo-Curing Process of Dental Resins with the NMR-MOUSE
}

\author{
Antonio Marchi Netto • Johannes Steinhaus • \\ Berenika Hausnerova • Bernhard Moeginger • \\ Bernhard Blümich
}

Received: 31 January 2013/Revised: 17 April 2013/Published online: 4 June 2013

(C) Springer-Verlag Wien 2013

\begin{abstract}
The photo-curing reaction of dental resins has been examined with unilateral nuclear magnetic resonance (NMR-MOUSE) allowing nondestructive high-resolution measurement of depth profiles as a function of time and space. The NMR signal is sensitive to both the monomer concentration and changes in molecular mobility. Upon irradiation with blue light, it first increases due to molecular mobility enhanced by the reaction heat and then decreases exponentially with the monomer concentration as the polymer signal is lost in the dead time of the instrument upon curing. The space and time dependence of the NMR signal can be described by the photo-polymerization reaction kinetics together with a heuristic approximation of the temperature dependence.
\end{abstract}

\section{Introduction}

Visible light curing (VLC) dental composites made of highly filled acrylic resins are successfully replacing amalgam fillings in tooth restoration during past three decades. These resins are polymerized in about 20-40 s using a photoinitiator (camphor quinone, CQ) amine accelerator system that is activated by an LED light

\footnotetext{
A. Marchi Netto · B. Blümich ( $₫)$

Institut für Technische und Makromolekulare Chemie, RWTH Aachen University, Worringerweg 1, 52074 Aachen, Germany

e-mail: bluemich@itmc.rwth-aachen.de

J. Steinhaus · B. Hausnerova

Centre of Polymer Systems, Department of Production Engineering,

Tomas Bata University in Zlín, Zlín, Czech Republic

J. Steinhaus · B. Moeginger

Department of Natural Sciences, Bonn-Rhein-Sieg University of Applied Sciences, Rheinbach, Germany
} 
curing unit (LCU) having a wavelength range of $440-480 \mathrm{~nm}$ and irradiances of 600-2,000 $\mathrm{mW} / \mathrm{cm}^{2}$, typically.

Besides polymerization shrinkage, biocompatibility, and wear resistance, one of the most frequently discussed issues in VLC resin composite development is the general question of how fast and how thick a light-cured material can be processed properly in dental applications [1-3]. Since the development of these materials a vast quantity of studies has investigated the curing kinetics [4-6], degree of cure (DC) [7, 8], and depth of cure (DoC) [8-10]. To characterize the curing process to optimize medical applications by predicting curing parameters like curing time and optimum light intensity as well as resin formulation, various test methods have been introduced and evaluated for time resolved monitoring of the curing process. The curing-enthalpy was analyzed with differential scanning calorimetry (DSC) [11, 12], the DC with Fourier transform infrared spectroscopy (FTIR) [3, 7, 8], the shrinkage strain with dilatometric devices [5, 6, 13], the shrinkage stress with stress-analyzers [7, 14, 15], the light transmission with radiometer measurements [4, 16], and the ionic conductivity with dielectric analysis (DEA) [16, 17]. All these methods provide time-dependent data and amongst others allow for kinetic modeling of the curing process and predicting the progress of the polymerization reaction.

Depth-dependent curing of VLC composites is usually examined through the depth of cure (DoC) parameter evaluated from FTIR, mechanical (hardness and scraping tests) and optical measurements [4, 8-11]. With hardness or scraping tests the DoC can only be obtained subsequent to the light-curing procedure. These results are always influenced by continuing diffusion-controlled polymerization and crosslinking as well as termination of radicals, which altogether govern the post-curing behavior and lead to time-dependent material properties. An optical or FTIR method allows measurement of the curing behavior of composite samples with different layer thicknesses; however, the DoC cannot be evaluated in a single bulky specimen. The kinetics of photo polymerization has been successfully studied through a $T_{2}$ relaxation analysis [18]. In this work, this phenomenon was studied by unilateral nuclear magnetic resonance not only with time resolution but also with depth resolution using the nuclear magnetic resonance (NMR)-MOUSE.

One of the most important features of a unilateral NMR sensor is its capacity to analyze objects non-destructively because the portable stray-field magnet does not need to be in a direct contact with the sample and does not restrict the sample size [19, 20]. In the characterization of polymers, unilateral NMR has been successfully used in the investigation of aging and cross-link density as a function of depth in elastomers and polyethylene [21-25]. The profile NMR-MOUSE ${ }^{\circledR}$ is made of four parallel magnets in a proper arrangement affirming that the NMR signal is collected from a thin and flat sensitive volume at a fixed distance above the magnet [26, 27]. A curing reaction is studied with this device, and the time- and depth-dependent NMR signals can be modeled with the polymerization kinetics of the reaction [28, 29].

\section{First-Order Kinetic Model of a Photo-Polymerization Reaction}

A polymerization reaction encompasses three stages: initiation, propagation and termination $[28,29]$. In the initiation stage the reaction is started, in our case by 
photo decomposition of an initiator into radical pairs, and these radicals react with the first monomer. In the propagation stage the macromolecular chains are formed by successive reaction with monomers until the reaction is terminated typically by combination, disproportionation, or lack of monomers. The reaction kinetics is as follows:

\subsection{Initiation}

The initiator I, in our case camphor quinone (CQ), is activated by light irradiation hv either to a singlet with a life time in the nano-second range or a triplet state with a life time of $50 \mathrm{~ms}$ [6]. Triplet-activated CQ molecules I* may react with accelerator molecules forming two radicals,

$$
\begin{gathered}
\mathrm{I}+\mathrm{h} v \rightarrow \mathrm{I}_{\text {triplet }}^{*} \\
\mathrm{I}_{\text {triplet }}^{*}+\mathrm{A} \rightarrow \mathrm{I}^{\cdot}+\mathrm{R}^{\cdot}
\end{gathered}
$$

The polymerization rate is determined by the accelerator radical $\mathrm{R}^{\bullet}$ reacting with the first monomer $\mathrm{M}$ as the $\mathrm{CQ}$ radical $\mathrm{I}^{\bullet}$ shows only little reactivity:

$$
\mathrm{R}^{\cdot}+\mathrm{M} \stackrel{k_{i}}{\longrightarrow} \mathrm{RM}^{\cdot}
$$

If $\varphi$ denotes the initiator efficiency, $I_{0}$ the intensity of the absorbed light at the surface which decomposes the initiator molecules, and $\alpha$ the extinction coefficient assuming an infinite reservoir of accelerator molecules, the depth depending rate of the two-step initiation reaction is given by [29]

$$
v_{\mathrm{i}}(\boldsymbol{d})=\boldsymbol{k}_{\mathrm{i}} \varphi \boldsymbol{I}_{0} \boldsymbol{t} \mathrm{e}^{-\alpha d}=-\frac{\mathrm{d}[\mathrm{M}]}{\mathrm{d} \boldsymbol{t}}
$$

with the depth $d$ of the reaction in the resin layer and the monomer concentration $[\mathrm{M}]$.

\subsection{Propagation}

$$
\mathrm{RM}_{n}^{\cdot}+\mathrm{M}_{1} \stackrel{k_{p}}{\longrightarrow} \mathrm{RM}_{n+1}^{\cdot}
$$

Approximating the rate of the bimolecular propagation reaction to be independent of the chain length, and denoting the concentration of growing chains by $\left[\mathrm{RM}^{\bullet}\right]$,

$$
v_{\mathrm{p}}=\boldsymbol{k}_{\mathrm{p}}[\mathrm{M}]\left[\mathrm{RM}^{\cdot}\right]=-\frac{\mathrm{d}[\mathrm{M}]}{\mathrm{d} \boldsymbol{t}}
$$

\subsection{Termination}

$$
\mathrm{RM}_{m}^{\cdot}+\mathrm{RM}_{m}^{\cdot} \stackrel{k_{t}}{\longrightarrow} \mathrm{M}_{m+m}
$$

The combined reaction rate for termination by combination and/or disproportionation is by convention written as 


$$
v_{\mathrm{t}}=2 \boldsymbol{k}_{\mathrm{t}}\left[\mathrm{RM}^{\bullet}\right]\left[\mathrm{RM}^{\bullet}\right] .
$$

In these reactions $k_{\mathrm{i}}, k_{\mathrm{p}}$, and $k_{\mathrm{t}}$ denote the reaction rate constants of the initiation, propagation, and termination steps. At constant temperature most monomers are consumed in the steady state when the initiation and termination reaction rates are identical, meaning $v_{\mathrm{i}}=v_{\mathrm{t}}$. Then the steady-state propagation rate of a radical photopolymerization is given by

$$
\frac{\mathrm{d}[\mathbf{M}]}{\mathrm{d} t}=-\boldsymbol{K} \sqrt{t}[\mathbf{M}]
$$

where

$$
K=k_{\mathrm{p}} \sqrt{\frac{k_{\mathrm{i}} \varphi I_{0}}{2 k_{\mathrm{t}}} \mathrm{e}^{-\alpha d}} .
$$

This leads to the time-dependent monomer concentration

$$
[\mathrm{M}](t)=[\mathbf{M}]_{0} e^{-\frac{2}{3}\left(\frac{t-t_{0}}{\tau}\right)^{3 / 2}}
$$

with the initial time $t_{0}$ and the initial monomer concentration $[\mathrm{M}]_{0}$, and

$$
\tau(\boldsymbol{d})=\boldsymbol{K}^{-2 / 3}(\boldsymbol{d})
$$

Note that Eq. (11) is not the stretched exponential function commonly known as the Kohlrausch-Williams-Watts (KWW) function [30], but rather the function proposed by Kohlrausch in his original work in 1854 [31].

\section{Low-Field NMR and NMR-MOUSE}

NMR is used as an analytical tool to study matter in a magnetic field under the impact of radio-frequency (rf) pulses. The samples under study must have a nuclear isotope with angular momentum (spin) different from zero which orients in a magnetic field [32]. In the present study hydrogen nuclei with spin $1 / 2$ were observed by relaxometry.

In a static magnetic field $\boldsymbol{B}_{0}$, the spins get aligned parallel to it. A radio frequency pulse with a magnetic field $\boldsymbol{B}_{1}$ perpendicular to $\boldsymbol{B}_{0}$ manipulates the nuclear magnetization, and the magnetization response to radio frequency pulses is analyzed. One of the most important NMR phenomena is the echo caused by constructive interference of magnetization components. A spin echo is generated by two rf pulses and observed at the echo time $t_{\mathrm{E}}$ after the first pulse, where $t_{\mathrm{E}}$ is twice the pulse separation. With the help of spin echoes, the transverse NMR relaxation time $T_{2}$ is determined.

NMR relaxometry concerns the measurement of the NMR relaxation times $T_{1}$ and $T_{2}$. The longitudinal relaxation time $T_{1}$ specifies the time needed for the magnetization to reach thermal equilibrium. The transverse relaxation time $T_{2}$ is the time with which the impulse response decays in a perfectly homogeneous magnetic field. When the field is inhomogeneous, the impulse response decays faster with the relaxation time $T_{2 \text { eff. }}^{*}$ Carr et al. [33, 34] proposed a multi-echo method to measure a train of echoes in 
inhomogeneous fields whose decay traces the decay of the impulse response in homogeneous fields in the absence of translational diffusion. Given the self-diffusion coefficient $D$, the decay of the magnetization signal $M(t)$ is described by

$$
\boldsymbol{M}_{x y}(\boldsymbol{t})=\boldsymbol{M}_{0} e^{-\left(\frac{1}{T_{2}}+\frac{1}{12} \gamma^{2} \boldsymbol{G}^{2} \boldsymbol{t}_{E}^{2} \boldsymbol{D}\right) t}=\boldsymbol{M}_{0} e^{-\frac{t}{T_{2 \mathrm{eff}}}}
$$

with the time lag between successive echoes $t_{\mathrm{E}}$, the gyromagnetic ratio characteristic of each isotope $\gamma$, the magnetic field gradient $G$. The characteristic decay constant of a Carr-Purcell-Meiboom-Gill (CPMG) echo train is called $T_{2 \text { eff }}$, and under low absolute values of $G, D$, and $t_{\mathrm{E}}$, it is well approximated by $T_{2}$.

\section{Experimental}

\subsection{Dental Resins}

Two commercial dental resin composites, "Arabesk Top" and "Grandio" (Voco $\mathrm{GmbH}$, Cuxhaven, Germany); with the standard color OA2 were investigated. They differ in their chemical composition and filler content (Arabesk $=23$ mass $-\%$ monomer, Grandio = 13 mass $-\%$ monomer). The curing process was photoactivated by applying blue light from a Celalux LED LCU with a maximum wavelength of $450 \mathrm{~nm}$.

\subsection{The Experimental Setup}

All NMR measurements were carried out with a stray field NMR sensor, the NMRMOUSE PM 5 (Magritek GmbH, Aachen, Germany) which collects NMR signal from a slice having a distance of $5 \mathrm{~mm}$ from the sensor surface. The NMR-MOUSE can be used to acquire depth profiles locally into the object by varying the distance between the NMR-MOUSE and the object. Coupled with a surface coil, which generates a $\boldsymbol{B}_{1}$ field perpendicular to the $\boldsymbol{B}_{0}$ field, the frequency of the NMRMOUSE ranges from 10 to $30 \mathrm{MHz}$ with gradients between 2 and $35 \mathrm{~T} / \mathrm{m}$ and a maximum range up $25 \mathrm{~mm}$ [35]. This NMR sensor was operated by a Bruker Minispec spectrometer at a Larmor frequency of $18.1 \mathrm{MHz}$ for ${ }^{1} \mathrm{H}$. An initial profile showed that the configuration provides $3 \mathrm{~mm}$ operational depth access with spacers suitably placed between magnet and coil of the NMR-MOUSE. The pulse length was set to $8 \mu \mathrm{s}$, the echo time was $32 \mu \mathrm{s}$, the acquisition window of the echo was $6 \mu \mathrm{s}$, and the recycle delay was adjusted to $0.15 \mathrm{~s}$. The samples studied had a thickness of either $3 \mathrm{~mm}$ allowing testing at four different positions or $4 \mathrm{~mm}$ to test six positions. In the following, the space scale is always the distance from the sample surface closest to the light source to the sensitive slice analyzed, set to 1.2, $1.7,2.2,2.7,3.3$, and $3.8 \mathrm{~mm}$.

In dentistry, a curing reaction takes less than $40 \mathrm{~s}$. This is too fast for the NMR analysis. Therefore, 24 common glass slides having a thickness of $1 \mathrm{~mm}$ were used as light attenuator, slowing down the curing time to about $10 \mathrm{~min}$. Fixing the number of scans to 128 , a $T_{2}$ measurement takes $25 \mathrm{~s}$. As it was not possible to stop 

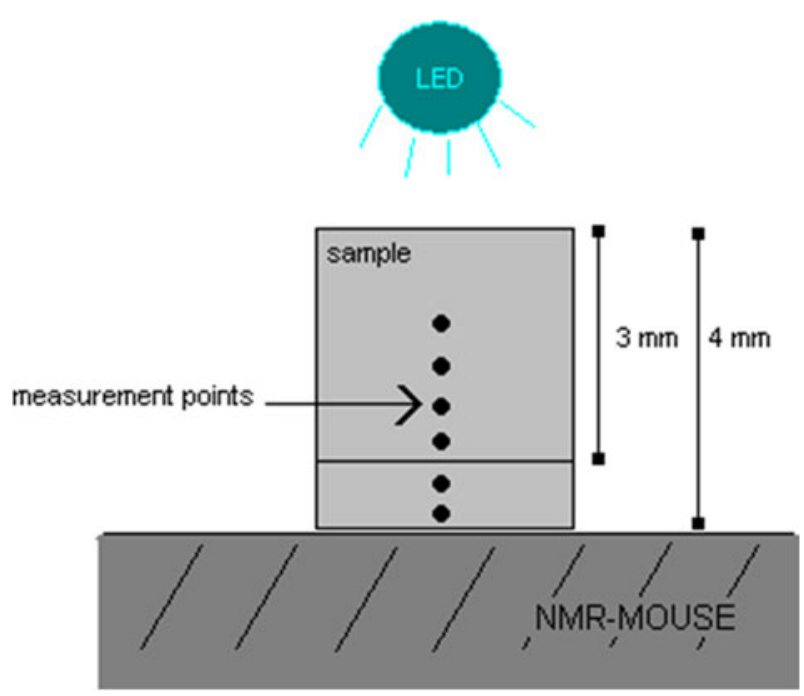

Fig. 1 NMR setup and positions of the sensitive slices in the sample

the photo-reaction, the $T_{2}$ measurements are an average of the signal changing during $25 \mathrm{~s}$. This approximation is acceptable when changes are weak, for example, during the stationary state of a photo-curing reaction, but introduces systematic errors when changes are more pronounced, like at the beginning or the end of the reaction. In such an experiment the NMR echoes arise only from the mobile monomers present in the sample while the signal from the solid polymer rapidly relaxes within about $15 \mu$ s during the dead time of the sensor.

In order to correlate the NMR-results with the temperature history of the sample in the different relevant layers during the curing process, corresponding measurements were performed collecting the temperature evolution with a thermocouple (type $\mathrm{K}, \varnothing 1 \mathrm{~mm}$ ) at measurement points similar to those of the NMR setup (Fig. 1). The sample temperature during polymerisation was collected for the first $140 \mathrm{~s}$ after the start of illumination of both materials (Arabesk and Grandio) each in the following layer depths of the sample: 2.15, 3.40, and $4.40 \mathrm{~mm}$. All measurements were performed three times.

\section{Results and Discussion}

\subsection{NMR Measurements}

Due to the short measurement time window of $25 \mathrm{~s}$, the CPMG decays have no good signal-to-noise ratio as illustrated in Fig. 2a (sample Arabesk, slice position at $1.2 \mathrm{~mm})$. In fits with exponential decays; the fit parameters "amplitude" and "relaxation time" show errors exceeding $30 \%$. Therefore, they could not be used to follow the reaction. On the other hand the echo sum has a sufficiently good signalto-noise ratio for that purpose. Figure $2 b$ and $c$ summarizes the echo sums of the 
Fig. 2 a Time dependent CPMG decays of the Arabesk sample, at slice position of $1.2 \mathrm{~mm}$. b Sums of the 64 first echoes in the CPMG detection trains at different depths versus the curing time by means of single-sided NMR for the Arabesk sample. c Sums of the 64 first echoes in the CPMG detection trains at different depths versus the curing time by means of single-sided NMR for the Grandio sample
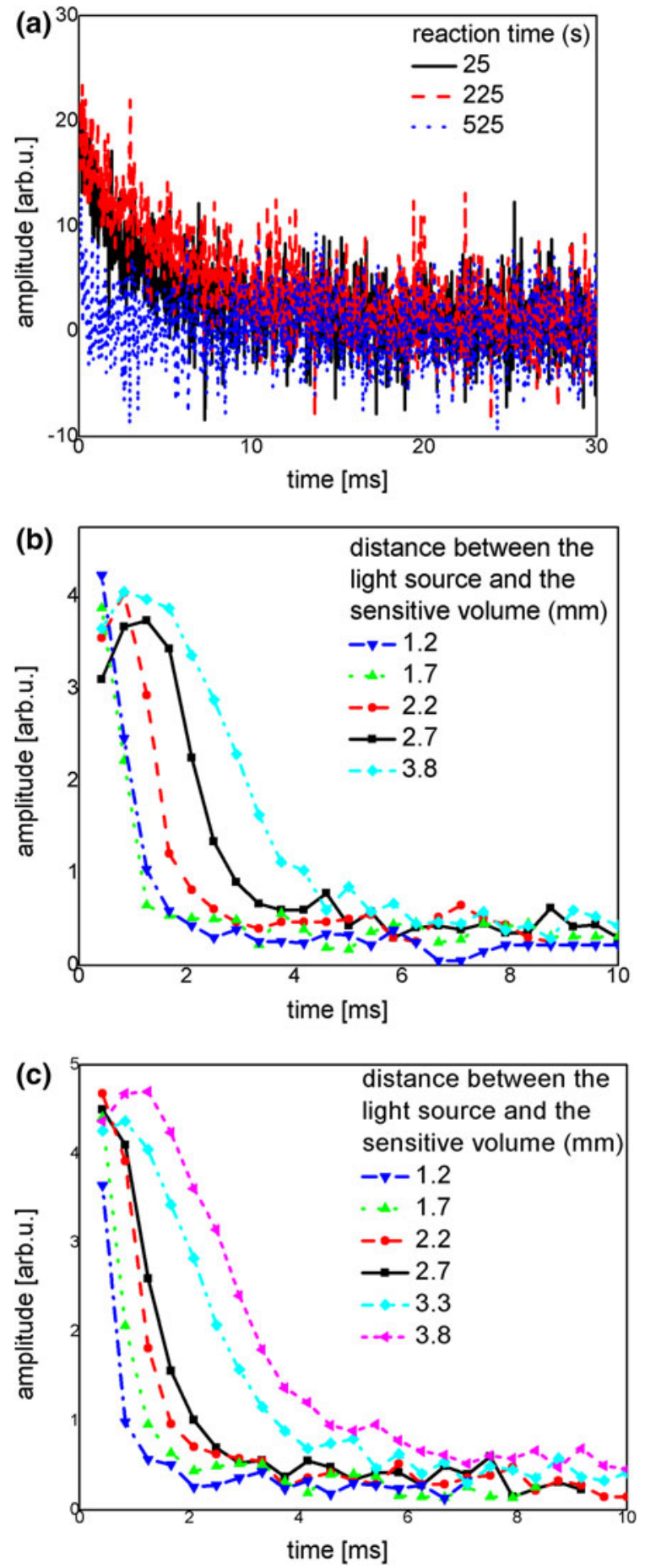
two dental resin composites as functions of time and depth corresponding to the distance from the light source.

Two different time periods can be discriminated in the curing curves depicted in Fig. $2 b$ and c: (1) an initiation phase, and (2) a curing phase. The duration of the initiation phase increases with increasing depth as the light, which initiates the reaction, is more attenuated. The subsequent curing process obeys Eq. (11) with the reaction rate constant which decreases with increasing distance from the light source. To describe the complete curing curves, two new terms were empirically included in Eq. (11): term 1 has a growing variance which takes account for the temperature change during the initiation phase, and term 2 represents an experimental offset $C$ yielding

$$
[\mathrm{M}](t)=[\mathrm{M}]_{0} \underbrace{\frac{2}{3}\left(\frac{t-t_{0}}{\tau}\right)^{3 / 2}}_{\text {term } 1} \mathbf{e}^{\left[-\frac{2}{3}\left(\frac{t-t_{0}}{\tau}\right)^{3 / 2}\right]}+\underbrace{C}_{\text {term } 2} .
$$

Differentiation of Eq. (14) provides the time when the NMR signal achieves its maximum value

$$
\boldsymbol{t}_{\mathrm{MAX}}=\left(\frac{3}{2}\right)^{2 / 3} \tau+\boldsymbol{t}_{0}
$$

Figure 3a shows examples of fits of the experimental data by Eq. (14) for the Arabesk sample at depths of 1.7 and $3.8 \mathrm{~mm}\left(R^{2}>0.98\right)$, with all parameters as variables. The spatial dependence of the maximum signal with the relative error bars is presented in Fig. 3b. It is shown below that a constant value for the initial monomer concentration $[\mathrm{M}]_{0}$ is found for all depths, and the reaction time constants $\tau$ depend exponentially on depth as expected from Eqs. (10), (12), and (14). The offset level increases with increasing depth.

\subsection{The Initiation Phase}

In the initial phase of the reaction (Fig. 2b, c), the NMR signal amplitude grows up to a maximum. This signal increase is attributed to the increase of molecular mobility from rising temperature. This means that less signal is lost in the dead time of the NMR-MOUSE. The effect of temperature was investigated in a series of experiments, where a temperature sensor was placed underneath samples of different thickness, and the temperature was monitored as a function of the curing time (Fig. 4). In these experiments the light intensity was the same as in the NMR experiments. The initial temperatures rise follows well the NMR signal rise. After reaching the maximum temperature the subsequent cooling is slow, and one can assume a constant temperature for the subsequent analysis.

\subsection{The Curing Phase}

In the curing phase, the decay of the echo sum shows the same time dependence as the monomer concentration in Eq. (14). The characteristic time constants $\tau(d)$ are related to the constant $K(d)$ according to Eq. (12). They both depend exponentially 
Fig. 3 a Signals selected for the Arabesk sample from Fig. $2 b$ and $c$ and fitted using Eq. (14). b Relation between the maximum of signal time and depth
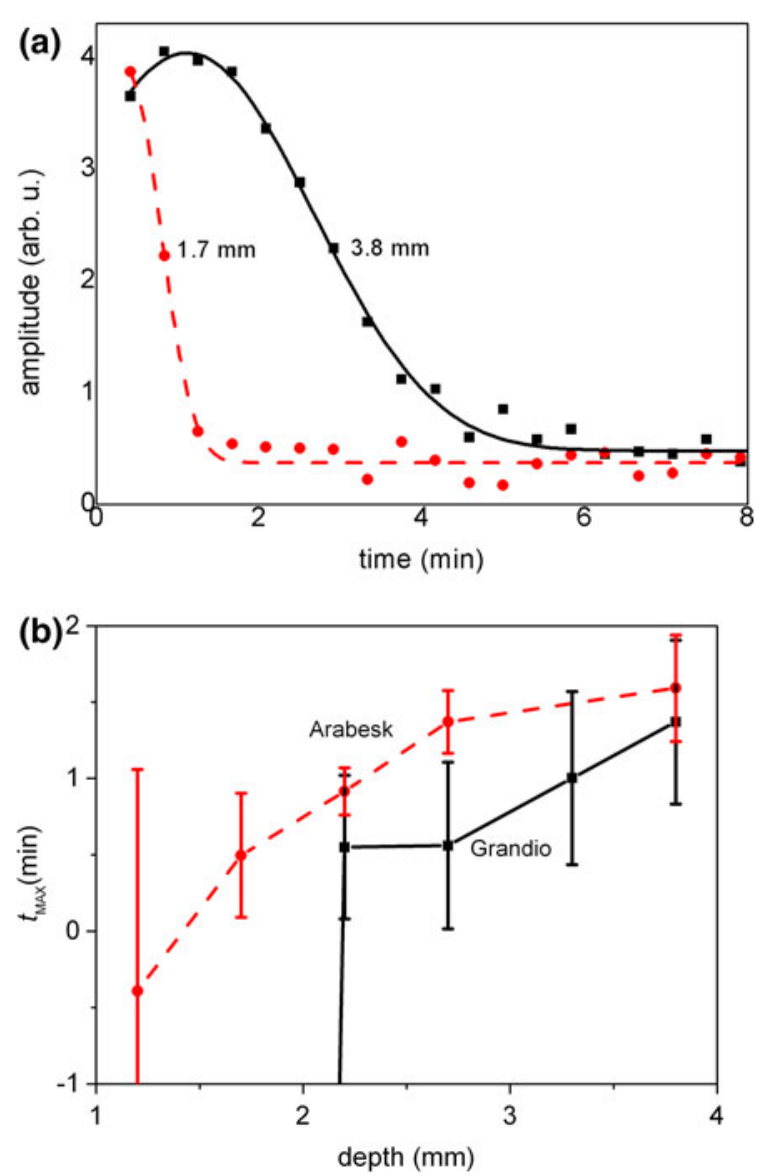

on the depth $d$ (Fig. 5). However, the characteristic time constants at low depths close to the surface do not follow the exponential trend due the absence of a signal maximum and some difficulties to follow the reaction at high reaction rates, with good accuracy. Therefore, the first points were discarded.

Figure 5 summarizes the decay and reaction speed constants of the magnetization during the steady state of the polymerization reaction as function of depth into the sample. The experimental reaction speed constants are in accordance with Eq. (14) and the associated decay constant is the extinction coefficient $\alpha$ (Table 1). For both Arabesk and Grandio, the extinction coefficients determined by NMR agree well with the ones directly determined by optical transmission measurements (OTE).

While reaction time $\tau$ and reaction rate constant $K$ approximately follow exponential laws at depth exceeding $2 \mathrm{~mm}$, their values at shallow depths are strongly impacted by fast temperature changes of the sample at these depths and the limited time resolution of the experiment. The same agreement applies to the time lags in Fig. 3b. These data points are, therefore, characterized as outliers and 
Fig. 4 Temperature as a function of reaction time for different sample thicknesses normalized to a common initial temperature. a Sample Arabesk. b Sample Grandio
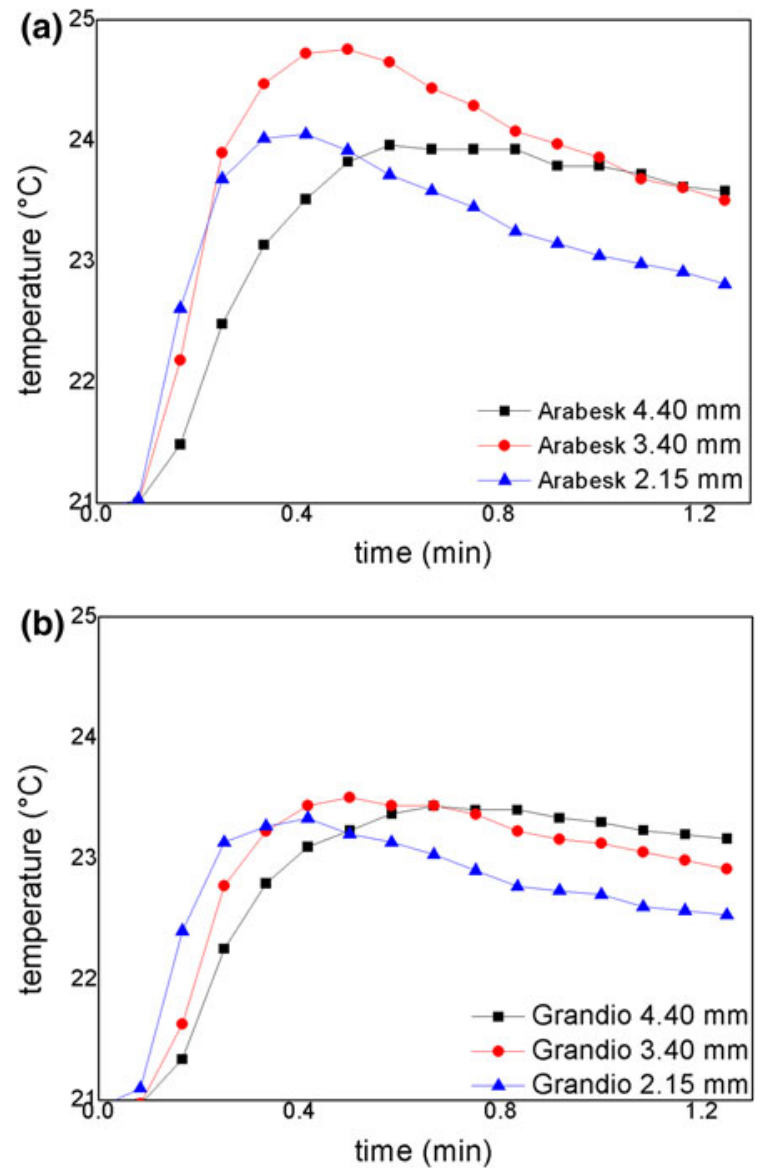

neglected in the determination of the extinction coefficient $\alpha$ from Eq. (10) (Table 1).

\section{Summary}

The NMR-MOUSE showed to be a useful tool for the analysis of slow photo-curing reactions of dental resin samples under small irradiation intensities as they occur in depths exceeding $2 \mathrm{~mm}$. By varying the distance between the NMR-MOUSE and the sample, the sensitive volume could be shifted across the thickness of the sample to follow the curing reaction at different depths going from light to dark curing of dental resins. A signal maximum was observed and attributed to the temperature increase caused by the exothermic reaction. Using an empirical equation the occurrence time of the temperature maximum was estimated. The experimental data were modeled with the textbook equations for first-order reaction kinetics of a photo initiated radical polymerization. 

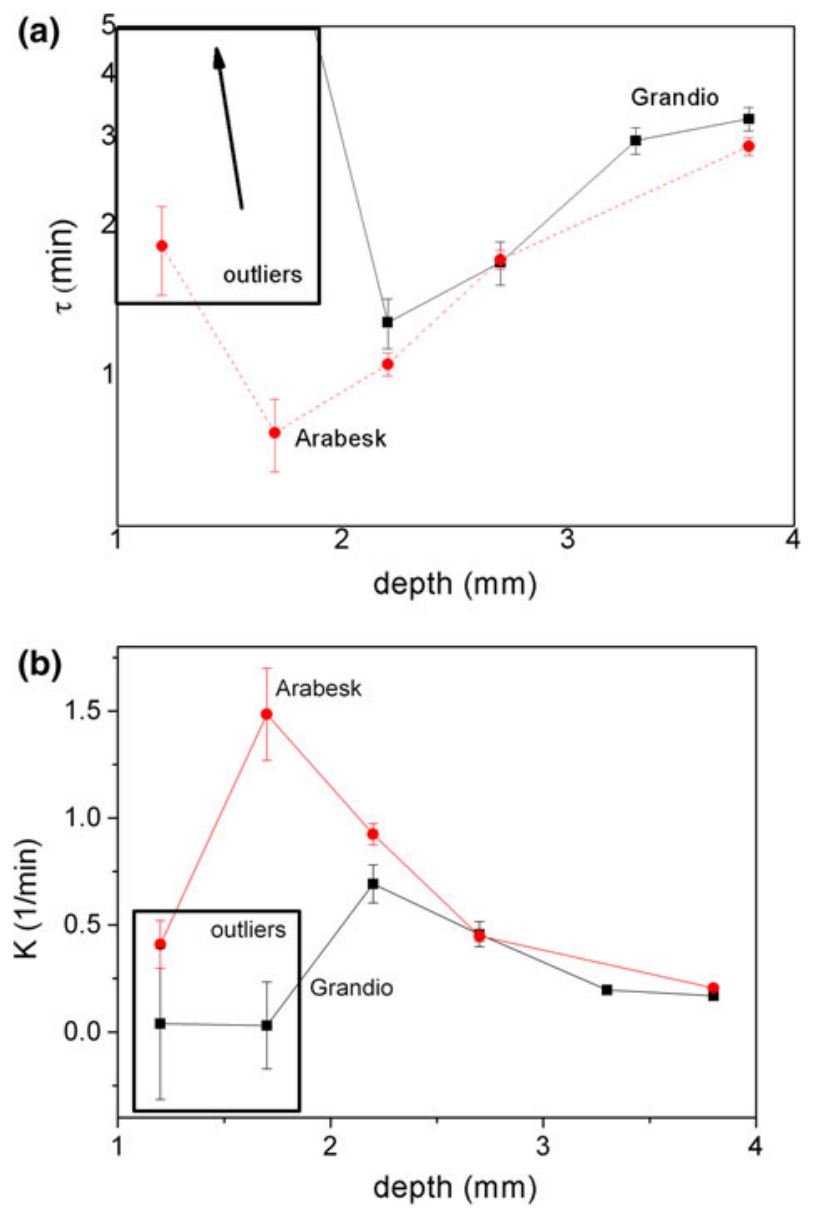

Fig. 5 Characteristic constant $\tau$ of reaction (a) and the reaction constant $K$ (b) at different depths extracted from Fig. 2. The outliers are indicated

Table 1 Extinction coefficients extracted from Fig. 5 and determined by optical transmission experiments (OTE)

\begin{tabular}{llr}
\hline Sample & $\alpha_{\mathrm{NMR}}\left(\mathrm{m}^{-3}\right)$ & $\alpha_{\mathrm{OTE}}\left(\mathrm{m}^{-3}\right)$ \\
\hline Arabesk & $0.7 \pm 0.1$ & $0.64 \pm 0.03$ \\
Grandio & $0.7 \pm 0.2$ & $0.60 \pm 0.03$ \\
\hline
\end{tabular}

It is concluded that the two analyzed resins polymerize on similar time scales. The relation proposed by Kohlrausch in 1854 was implemented in the kinetic formula to account for the initial temperature rise of the material at the beginning of the photo polymerization time. In the proposed form the resultant semi-empirical expression fits well the time dependence of the curing reaction. The depth dependence of the reaction kinetics is in accordance with the Lambert-Beer law. 
The photo polymerization of the dental resins thus can be modeled as a function of reaction time and depth for the benefit of assisting the optimization of resin formulations and curing procedures.

These results will help to gain insight into the curing kinetics for low radical concentrations as it is the case at the bottom of thick dental fillings. Furthermore, they allow to trace the aging behavior of incompletely light-cured fillings. Both issues are of high interest for dental restorations because treatment times are to be kept as short as possible for the patients' convenience and economic benefit.

Acknowledgments The author A.M.N. thanks the Brazilian National Council for Scientific and Technological Development (CNPq) for financial support (Grant No. 290010/2009-8). The author B.M. thanks the Federal Republic of Germany, Ministry of Education and Research for financial support due to the FHProfUnt project (Grant No. 17081X10). The author B.H. acknowledges the support of Operational Program Research and Development for Innovations co-funded by the European Regional Development Fund (ERDF) and national budget of Czech Republic, within the framework of project Centre of Polymer Systems (reg. number: CZ.1.05/2.1.00/03.0111).

\section{References}

1. F.A. Rueggeberg, Dent. Mater. 27, 39-52 (2011)

2. N. Moszner, U. Salz, Prog. Polym. Sci. 26, 535-576 (2001)

3. L.G. Lovell, K.A. Berchtold, J.E. Elliott, H. Lu, C.N. Bowman, Polym. Adv. Technol. 12, 335-345 (2001)

4. Y.C. Chen, J.L. Ferracane, S.A. Prahl, Dent. Mater. 21, 1075-1086 (2005)

5. D.C. Watts, A.J. Cash, Dent. Mater. 7, 281-287 (1991)

6. D.C. Watts, Dent. Mater. 21, 27-35 (2005)

7. I. Sideridou, V. Tserki, G. Papanastasiou, Biomaterials 23, 1819-1829 (2002)

8. L. Musanje, J.L. Ferracane, R.L. Sakaguchi, Dent. Mater. 25, 994-1000 (2009)

9. J.L. Ferracane, P. Aday, H. Matsumoto, V.A. Marker, Dent. Mater. 2, 80-84 (1986)

10. R.B.T. Price, C.A. Felix, P. Andreou, Biomaterials 26, 2631-2641 (2005)

11. J. Vaidyanathan, T.K. Vaidyanathan, Y. Wang, T. Viswanadhan, J. Oral Rehabil. 19, 49-64 (1992)

12. M. Rosentritt, M. Behr, A. Leibrock, G. Handel, J. Mater. Sci. 10, 91-98 (1999)

13. D.C. Watts, A.S. Marouf, Dent. Mater. 16, 447-451 (2000)

14. J.R. Condon, J.L. Ferracane, J. Am. Dent. Assoc. 131, 497-503 (2000)

15. M. Cadenaro, M. Biasotto, N. Scuor, L. Breschi, C.L. Davidson, R. Di Lenarda, Dent. Mater. 24, 681-685 (2008)

16. M. Rosentritt, A.C. Shortall, W.M. Palin, Dent. Mater. 26, 565-570 (2010)

17. J. Steinhaus, B. Moeginger, M. Grossgarten, B. Hausnerova, Mater. Eng. 18, 28-33 (2011)

18. V. Litvinov, A.A. Dias, Macromol. Symp. 230, 20-25 (2005)

19. J. Perlo, F. Casanova, B. Blümich, J. Mag. Res. 176, 64-70 (2005)

20. F. Presciutti, J. Perlo, F. Casanova, S. Gloggler, C. Miliani, B. Blümich, B.G. Brunetti, Appl. Phys. Lett. 93, 033505 (2008)

21. N.O. Goga, D.E. Demco, J. Kolz, R. Ferencz, A. Haber, F. Casanova, B. Blümich, J. Magn. Reson. 192(1), 1-7 (2008)

22. J. Kolz, J. Martins, K. Kremer, T. Mang, B. Blumich, Kautschuk Gummi Kunst. 60, 179-183 (2007)

23. B. Blümich, F. Casanova, A. Buda, K. Kremer, T. Wegener, Acta. Phys. Pol. A 1, 13-23 (2005)

24. B. Blümich, F. Casanova, J. Perlo, S. Anferova, V. Anferov, K. Kremer, N. Goga, K. Kupferschläger, M. Adams, Magn. Reson. Imag. 23, 197-201 (2005)

25. M.A. Vargas, K. Sachsenheimer, G. Guthausen, Pol. Tes. 31(1), 127-135 (2012)

26. G. Eidmann, R. Savelsberg, P. Blümler, B. Blümich, J. Magn. Reson. A 122, 104-109 (1996)

27. B. Blümich, P. Blümler, G. Eidmann, A. Guthausen, R. Haken, U. Schmitz, K. Saito, G. Zimmer, Magn. Reson. Imag. 16, 479-484 (1998) 
28. J.M.G. Cowie, V. Arrighi, Polymers: Chemistry and Physics of Modern Materials, 3rd edn. (CRC Press, USA, 2007)

29. P.C. Hiemenz, Polymer Chemistry (Marcel Dekker, New York, 1984)

30. F. Kohlrausch, Pogg. Ann. Phys. 119, 352 (1863)

31. F. Kohlrausch, Pogg. Ann. Phys. 167, 56-82 (1854)

32. E. Fukushuma, S.B.W. Roeder, Experimental Pulse NMR (Addison-Wesley, MA, 1981)

33. H.Y. Carr, E.M. Purcell, Phys. Rev. 94, 630-638 (1954)

34. S. Meiboom, D. Gill, Rev. Sci. Instrum. 29, 688-691 (1958)

35. http://www.act-aachen.com/profile_MOUSE.html. Accessed 20 Aug 2012 